\title{
Rashba interaction in quantum wires with in-plane magnetic fields
}

\author{
Llorenç Serra, ${ }^{1,2}$ David Sánchez, ${ }^{1}$ and Rosa López ${ }^{1}$ \\ ${ }^{1}$ Departament de Física, Universitat de les Illes Balears, E-07122 Palma de Mallorca, Spain \\ ${ }^{2}$ Institut Mediterrani d'Estudis Avançats IMEDEA (CSIC-UIB), E-07122 Palma de Mallorca, Spain
}

(Received 27 April 2005; revised manuscript received 17 June 2005; published 7 December 2005)

\begin{abstract}
We analyze the spectral and transport properties of ballistic quasi-one-dimensional systems in the presence of spin-orbit coupling and in-plane magnetic fields. Our results demonstrate that Rashba precession and intersubband coupling must be treated on equal footing for wave vectors near the magnetic-field-induced gaps. We find that intersubband coupling limits the occurrence of negative effective masses at the gap edges and modifies the linear conductance curves in the strong-coupling limit. The effect of the magnetic field on the spin-textured orientation of the wire magnetization is discussed.
\end{abstract}

DOI: 10.1103/PhysRevB.72.235309

PACS number(s): 73.63.Nm, 71.70.Ej, 72.25.Dc

\section{INTRODUCTION}

Controllable manipulation of electron spins with electric fields (gates) is a central requirement of spintronic devices. Semiconductor heterostructures offer the possibility of electric control of spins through intrinsic spin-orbit interactions. A major contribution to spin-orbit effects in two-dimensional (2D) electron gases of narrow-gap semiconductors originates from the macroscopic electric field confining the electron gas. ${ }^{1}$ This implies an asymmetry in the quantum-well potential (the Rashba effect), which can be further tuned with gate voltages. The resulting spin-orbit coupling has been demonstrated in experiments. ${ }^{2-4}$

In this paper, we are concerned with the Rashba interaction in ballistic quantum wires. Interestingly, these systems have been proposed as basic elements for practical applications such as spin-dependent field-effect transistors ${ }^{5}$ and spin filters ${ }^{6}$ and have been also considered as detectors of entangled pairs of electrons ${ }^{7}$ and of the hyperfine nuclear spin dynamics. ${ }^{8}$ The Rashba interaction is described by the Hamiltonian,

$$
\mathcal{H}_{R}=-\frac{\alpha_{1}}{\hbar} p_{x} \sigma_{y}+\frac{\alpha_{2}}{\hbar} p_{y} \sigma_{x}
$$

where $\vec{p}=\left(p_{x}, p_{y}\right)$ is the linear momentum and $\sigma_{x}, \sigma_{y}$ are Pauli matrices. Spin transistors exploit the Rashba-induced precession of spins ( $\alpha_{1}$ term for transport along the $x$ direction). However, in Eq. (1) there is an additional term, proportional to $\alpha_{2}$, which mixes nearest subbands with opposite spins and induces anticrossings at the degeneracy points of the wire's energy spectrum. ${ }^{9,10}$ Obviously, in a device one has $\alpha_{1}=\alpha_{2}$, but it is of conceptual interest to distinguish in Eq. (1) between the two contributions (see below).

When an in-plane magnetic field is externally applied or it arises from ferromagnetic leads attached to the wire, the Zeeman splitting opens gaps in the wire spectrum, ${ }^{6,8,11-13}$ strongly affecting the transport properties. It also influences the spin dynamics since, whereas the Rashba precession randomizes the spin direction (a common spin quantization axis can be defined only for large wave vectors), the magnetic field tends to align the spins parallel to it. Many works neglect ${ }^{8,11-13}$ the effect of Rashba intersubband coupling
(RIC) in quantum wires in the presence of in-plane magnetic fields. Here we demonstrate that both Rashba precession and intersubband coupling must be treated on equal footing for wave vectors near the subband gaps. We find that RIC hinders the formation of subband maxima, smoothing the gap edges and, as a consequence, strongly affecting the conductance steps. This conclusion is most relevant to the strongcoupling limit, when spin-orbit and confinement energy scales are of the same order of magnitude. Section II presents the model and analyzes its characteristic energy subbands. In Sec. III we show the spin expectation values and magnetization distributions while Sec. IV focuses on the linear conductance and, finally, the conclusions are contained in Sec. V.

\section{HAMILTONIAN AND ENERGY SPECTRUM}

We consider a quantum wire formed when a $2 \mathrm{D}$ gas is further confined in one direction (see Fig. 1). The confinement is assumed parabolic in the $y$ direction, $\mathcal{H}_{\text {conf }}$ $=m \omega_{0}^{2} y^{2} / 2$, giving a wire orientation along $x$. An in-plane magnetic field $\vec{B}=(B \cos \theta, B \sin \theta)$ acts through the Zeeman Hamiltonian $\mathcal{H}_{Z}=g \mu_{B} B\left(\cos \theta \sigma_{x}+\sin \theta \sigma_{y}\right) / 2$. Orbital magnetic effects are absent in this geometry since they arise only from perpendicular fields. ${ }^{4,14,15}$ Adding all contributions, the resulting quasi-1D Hamiltonian reads

$$
\mathcal{H}=\left(p_{x}^{2}+p_{y}^{2}\right) / 2 m+\mathcal{H}_{\text {conf }}+\mathcal{H}_{Z}+\mathcal{H}_{R}
$$

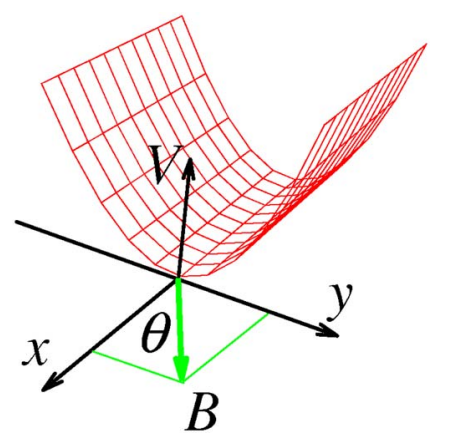

FIG. 1. (Color online) Schematic representation of the wire potential $\mathcal{H}_{\text {conf }} \equiv V(y)=m \omega_{0}^{2} y^{2} / 2$ and magnetic-field orientation considered in this work. 
Since the system is translationally invariant along $x$, the wave function dependence on this variable is $e^{i k x}$, with $k$ the propagation wave vector. It is then useful to recast the Hamiltonian for a given wave vector, $\mathcal{H}_{k}$, in dimensionless form: ${ }^{15}$

$$
\begin{aligned}
\frac{\mathcal{H}_{k}}{\hbar \omega_{0}}= & \left(\hat{n}_{k}+\frac{1}{2}\right)+\frac{1}{2}\left(\frac{l_{0}}{l_{Z}}\right)^{2}\left(\cos \theta \sigma_{x}+\sin \theta \sigma_{y}\right)+\frac{\left(k l_{0}\right)^{2}}{2} \\
& -\frac{l_{0}}{2 l_{1}}\left(k l_{0}\right) \sigma_{y}+\frac{i l_{0}}{2 \sqrt{2} l_{2}}\left(\hat{a}_{k}^{\dagger}-\hat{a}_{k}\right) \sigma_{x},
\end{aligned}
$$

with the characteristic lengths $l_{0}=\sqrt{\hbar / m \omega_{0}}$ (confinement), $l_{Z}=\sqrt{\hbar^{2} / m g \mu_{B} B} \quad$ (Zeeman coupling, $\left.g>0\right),{ }^{16}$ and $l_{i}$ $=\hbar^{2} / 2 m \alpha_{i}(i \in 1,2$, Rashba interaction). We use in Eq. (3) the bosonic operator $a_{k}\left(a_{k}^{\dagger}\right)$ which lowers (raises) a subband index for fixed $k$; thereby, $\hat{n}_{k}=a_{k}^{\dagger} a_{k}$ is the number operator. The third term on the right-hand side of Eq. (3) describes the free propagation motion in the $x$ direction whereas the forth and fifth contributions correspond to Rashba precession $\left(l_{1}\right)$ and to RIC $\left(l_{2}\right)$, respectively. The $l_{1}$ Rashba coupling induces subband spin splitting whereas it is clear from Eq. (3) that the $l_{2}$ term couples adjacent subbands with opposite spins. In writing $\mathcal{H}_{k}$ we have omitted the Coulomb interaction between the electrons since its effect can be taken into account, at least in part, via a renormalized Rashba coefficient. ${ }^{17}$

Due to the presence of RIC, $\mathcal{H}_{k}$ is not diagonalizable except for a special wave vector (see below). Yet it is easy to show that for fields parallel to the wire the two Rashba terms contribute equally. Without a Rashba interaction the energy dispersion of a given subband is $E_{n k \eta}^{(0)}=(n+1 / 2)+\left(k l_{0}\right)^{2} / 2$ $+\eta\left(l_{0} / l_{Z}\right)^{2} / 2$ with $n=0,1,2, \ldots$ and $\eta= \pm 1$. We find the energy correction to first order in the Rashba couplings:

$$
E_{n k \eta}^{(1)}=-\eta \frac{1}{2}\left(k l_{0}\right) \frac{l_{0}}{l_{1}} \sin \theta .
$$

Equation (4) already shows two noticeable features: (i) When $\theta \neq 0$ there is a Rashba-induced splitting proportional to $k$, well known from studies of spin-orbit effects at $B=0$ (see, e.g., Ref. 18, which combines with the Zeeman splitting to yield multiple subband crossings, and (ii) RIC (the $l_{2}$ term) does not contribute to first order but for $\theta=0$ the Rashba precession $\left(l_{1}\right)$ term is also zero. This implies that when $\theta$ $=0$ both Rashba terms contribute to second order in the couplings. The full second-order correction reads

$$
\begin{aligned}
E_{n k \eta}^{(2)}= & \eta \frac{l_{0}^{2}}{4 l_{1}^{2}}\left(k l_{0}\right)^{2} \frac{l_{Z}^{2}}{l_{0}^{2}} \cos ^{2} \theta \\
& -\frac{l_{0}^{2}}{8 l_{2}^{2}}\left[1+\sin ^{2} \theta \frac{\left(l_{0} / l_{Z}\right)^{4}+\eta(2 n+1)\left(l_{0} / l_{Z}\right)^{2}}{1-\left(l_{0} / l_{Z}\right)^{4}}\right] .
\end{aligned}
$$

Adding all corrections one has $E_{n k \eta} \simeq E_{n k \eta}^{(0)}+E_{n k \eta}^{(1)}+E_{n k \eta}^{(2)}$, which is valid for $l_{0}, l_{Z} \ll l_{1}, l_{2}$ and $l_{0} \neq l_{Z}$. It should be emphasized that in the above perturbative analysis we have taken as expansion parameters $l_{0} / l_{1}$ and $l_{0} / l_{2}$, assuming that the remaining factors of the two Rashba terms are similari.e., that $k l_{0} \simeq 1$. Obviously, in the limit of very large $k$ the Rashba precession term, proportional to $k l_{0}$, will clearly dominate over the RIC.

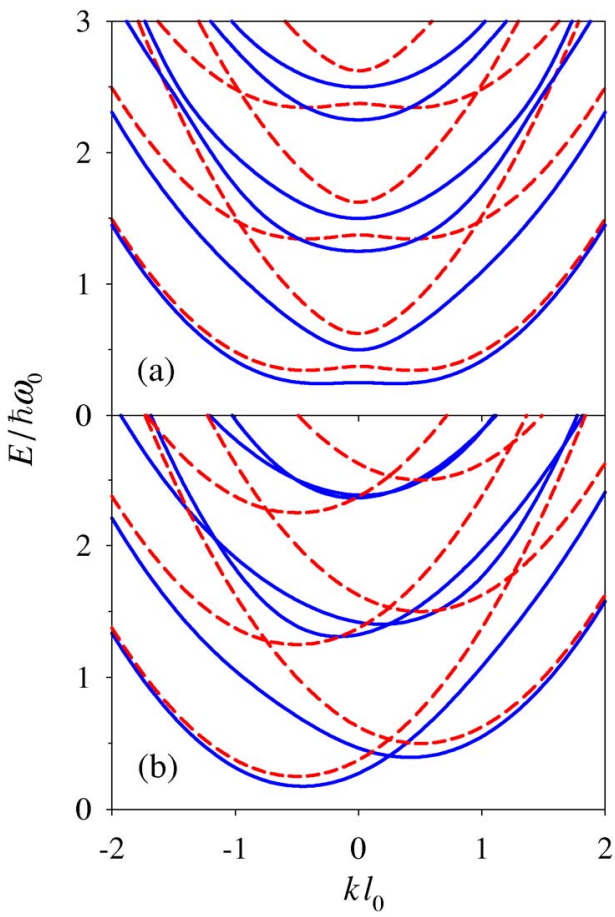

FIG. 2. (Color online) (a) Dispersion relation for $\theta=0, l_{Z}=2 l_{0}$, and $l_{1}=l_{0}$. The solid line corresponds to the case where the Rashba intersubband coupling is fully included $\left(l_{2}=l_{1}\right)$ while the dashed line shows the case without it $\left(l_{2}=\infty\right)$. (b) Same as (a) for $\theta=\pi / 2$.

Further progress is made if we diagonalize the total Hamiltonian in the absence of RIC-i.e., setting $l_{2} \rightarrow \infty$ in Eq. (3). The exact eigenstates and eigenenergies (in $\hbar \omega_{0}$ units)

$$
\begin{gathered}
\psi_{n k \eta}(x, y)=\frac{e^{i k x}}{\sqrt{2}} \phi_{n}(y)\left[e^{i \Omega_{k} / 2}|\uparrow\rangle+\eta e^{-i \Omega_{k} / 2}|\downarrow\rangle\right], \\
E_{n k \eta}=\left(n+\frac{1}{2}\right)+\frac{1}{2}\left(k l_{0}\right)^{2}+\eta\left|z_{k}\right|,
\end{gathered}
$$

are labeled, as before, with three quantum numbers: the propagation wave vector $k$, the index $n$ of the corresponding 1D harmonic oscillator wave function $\phi_{n}(y)$, and the branchsplitting quantum index $\eta= \pm 1$. The notation has been simplified by defining the complex number $z_{k}=\left(l_{0} / l_{Z}\right)^{2} e^{-i \theta} / 2$ $+i\left(k l_{0} / 2\right)\left(l_{0} / l_{1}\right)$ and its argument $\Omega_{k}=\operatorname{Arg}\left[z_{k}\right]$. This complex quantity takes into account the relative importance of Zeeman and Rashba precession energies, as can be seen more clearly when rewriting it like $z_{k}=\left[\left(g \mu_{B} B / 2\right) e^{-i \theta}+i \alpha_{1} k\right] / \hbar \omega_{0}$.

The energy spectrum of Eq. (7), for $\theta=0$ and $\theta=\pi / 2$, is plotted with dashed lines in Figs. 2(a) and 2(b) for $l_{Z}=2 l_{0}$ and strong spin-orbit $l_{1}=l_{0}$ (taking $\alpha \approx 10 \mathrm{meV} \mathrm{nm}$ and InAs parameters the above values would correspond to $\hbar \omega_{0}$ $\approx 0.2 \mathrm{meV}$ and $B \approx 0.1 \mathrm{~T}$, which can be achieved experimentally). ${ }^{4}$ For both angles the spectrum shows energy crossings between subbands with $n \neq n^{\prime}$ and opposite $\eta$. For $\theta=\pi / 2$ there are additional crossing points for $k l_{0} \approx 0.2$ between subbands of opposite $\eta$ and equal $n$. Noticeably, for $\theta=0$ the Zeeman field produces gaps at $k=0$ and around that 
point the spectrum shows local maxima for the $\eta=-1$ subbands. From Eq. (7) we see that a local maximum (minimum) occurs at the $\eta=-1$ subbands when $l_{Z}>\sqrt{2} l_{1}\left(l_{Z}\right.$ $\left.<\sqrt{2} l_{1}\right)$. Thus, changing the magnetic field affects dramatically the spectrum and, as discussed below, the transport properties.

Solid lines in Figs. 2(a) and 2(b) show the effect of RIC. We have obtained the spectrum with a direct numerical diagonalization of Eq. (3) in the basis of Eq. (6), truncating to a large enough $n$. The intersubband matrix elements of $\mathcal{H}_{\alpha_{2}}$ $\equiv\left(i l_{0} / 2 \sqrt{2} l_{2}\right)\left(a^{\dagger}-a\right) \sigma_{x}$ read

$$
\begin{aligned}
\left\langle n k \eta\left|\mathcal{H}_{\alpha_{2}}\right| n^{\prime} k^{\prime} \eta^{\prime}\right\rangle= & \frac{i}{4 \sqrt{2}} \frac{l_{0}}{l_{2}} \delta_{k, k^{\prime}}\left(\eta e^{i \Omega_{k}}+\eta^{\prime} e^{-i \Omega_{k}}\right) \\
& \times\left(\delta_{n^{\prime}, n-1} \sqrt{n}-\delta_{n^{\prime}, n+1} \sqrt{n+1}\right) .
\end{aligned}
$$

As an alternative method, we have also discretized $\mathcal{H}_{k}$ in real space with finite differences and diagonalized the resulting matrix. Both methods give identical results. When RIC is fully included we find that the crossings of different- $n$ subbands (dashed lines) become anticrossings (solid lines) as expected. Importantly, for $\theta=0$ and high-energy subbands RIC converts the maxima into minima [see, e.g., the second and third subbands in Fig 2(a)].

Figure 2 also shows a conspicuous downward shift of the solid lines with respect to the dashed ones for $k$ values close to the central gaps (for $\theta=0$ ) or degeneracy points (for $\theta$ $=\pi / 2$ ). This can be easily explained by noting that the minimum gap condition for $\theta \neq \pi / 2$ or crossing point for $\theta$ $=\pi / 2$ is given by $\operatorname{Im}\left[z_{k_{g}}\right]=0$ with solution $k_{g}=\left(l_{1} / l_{Z}^{2}\right) \sin \theta$. When this occurs the Hamiltonian (3) can be exactly solved since $\mathcal{H}_{\alpha_{2}}$ does not couple states with different $\eta$ indices [see Eq. (8) and note that $\left.\eta+\eta^{\prime}=2 \eta \delta_{\eta \eta^{\prime}}\right]$. The Hamiltonian for each $\eta$,

$$
\mathcal{H}_{\eta}=\frac{\hbar^{2} k_{g}^{2}}{2 m}+\frac{\left(p_{y}+\eta m \alpha_{2} / \hbar\right)^{2}}{2 m}+\frac{1}{2} m \omega_{0} y^{2}+\eta\left|z_{k_{g}}\right|-\frac{\alpha_{2}^{2} m}{2 \hbar^{2}},
$$

represents a harmonic oscillator with a shifted transverse momentum and a global negative energy constant. As a result, the exact states at the minimum gap are those of Eq. (6) shifted in momentum, $e^{-i \eta m \alpha_{2} y / \hbar^{2}} \psi_{n k_{g} \eta}(x, y)$, and the exact eigenenergies show a rigid shift $E_{n k_{g}} \eta-l_{0}^{2} / 8 l_{2}^{2}{ }^{2}{ }^{19}$

For $\theta=\pi / 2$ [Fig. 2(b)] we find new energy degeneracies entirely due to the presence of RIC in addition to the anticrossings similar to the $\theta=0$ case. For $0<\theta<\pi / 2$ there is a local minimum or maximum at $k_{g}$ depending on the size of $\alpha_{2}$ with respect to $\alpha_{1}$ and $B$. Our results, thus, demonstrate that including intersubband coupling is essential in any theory of strong Rashba interaction in quantum wires in the presence of magnetic fields and for $k$ values near the subband gaps.

To see how sensitive the local maxima are to the effect of RIC we calculate the second-order correction in $\mathcal{H}_{\alpha_{2}}$ to the energy spectrum at $\theta=0$ and then find the dimensionless effective mass at the extremum of the $n \eta$ branch, $m / m_{n \eta}^{*}$ $=\partial^{2} E_{n k \eta} /\left.\partial\left(k l_{0}\right)^{2}\right|_{k=k_{g}}$. The sign of $m^{*}$ determines the character of the extremum, electron like $\left(m^{*}>0\right)$ or hole like $\left(m^{*}\right.$ $<0)$ :

$$
\frac{m}{m_{n \eta}^{*}}=1+\frac{\eta}{2}\left(\frac{l_{Z}}{l_{1}}\right)^{2}\left[1-\frac{1}{2}\left(\frac{l_{0}}{l_{2}}\right)^{2} \frac{1+2 n+\sqrt{2} \eta\left(l_{0} / l_{Z}\right)}{1-2\left(l_{0} / l_{Z}\right)^{2}}\right] .
$$

For $\alpha_{2}=0$ and large $\alpha_{1}\left(l_{1}<l_{Z} / \sqrt{2}\right), m_{n \eta}^{*}$ becomes negative for $\eta=-1$ subbands, leading to a maximum in the energy spectrum, in agreement with our previous conclusions. However, for strong enough RIC the effective mass sign is reversed. We emphasize that this is an effect purely due to the RIC and it is even stronger for increasing $n$, confirming the numerical results of Fig. 2(a). Equation (10) is a perturbative result and works well provided the confinement is the smallest length scale $\left(l_{0} \ll l_{Z}, l_{1}, l_{2}\right)$. Only for very wide wires $\left(l_{1}, l_{2}, l_{Z} \ll l_{0}\right)$ we numerically recover maxima $\left(m_{n \eta}^{*}<0\right)$.

\section{SPIN ORIENTATION AND MAGNETIZATION}

In general, when both Rashba terms are present spin is not a good quantum number and we find spin textures, with the spin direction depending on $k$ and the wire transversal coordinate $y$. On the contrary, if RIC is neglected, the states are proper eigenspinors even in the presence of an in-plane magnetic field, ${ }^{11}$ as seen from Eq. (6). Therefore, the existence of clear spin textures is a signature of the RIC term. The local spin components for the lowest subband at three different propagation momenta are shown in the upper plots of Fig. 3. While all in-plane spins are essentially collinear, a sizable $z$ component, similar in magnitude to the in-plane one, precludes the definition of a common spin axis when $k l_{0} \neq 0$ and thus shows the importance of RIC. The local $z$ magnetization in real space $\left\langle\sigma_{z}(y)\right\rangle$ is antisymmetric in $y$, leading to a vanishing integrated $\left\langle\sigma_{z}\right\rangle$ (Ref. 20) and giving rise to spin accumulations at the wire edges which are reminiscent of the intrinsic spin Hall effect, ${ }^{21}$ but here the effect arises in a confined system. ${ }^{22}$

There are exceptional cases where the spin is well defined even in the presence of both a magnetic field and RIC. For $\left|k l_{0}\right| \gg 1$ one always finds asymptotic eigenstates of $\sigma_{y},{ }^{20} \mathrm{ir}-$ respectively of the magnetic-field orientation $\theta$, as seen in the lower plot of Fig. 3. For states with large negative (positive) $k$ the spin is $\left\langle\sigma_{y}\right\rangle=-1(+1)$. Importantly, for $k=0$ (the minimum gap point for $\theta=0$ ) we numerically find $\left\langle\sigma_{x}\right\rangle=\eta$, regardless of the strengths of the magnetic fields and intersubband coupling. This is clear from Eq. (9), which shows that the spinors are eigenstates of $\sigma_{x}$ at the minimum gap momentum. Therefore, there is always a given wave vector $k_{g}=\left(l_{1} / l_{Z}^{2}\right) \sin \theta$, satisfying $\Omega_{k_{g}}=0$, for which the propagation direction is also the spin quantization axis. Away from this special point, $\sigma_{x}$ tends to zero (see Fig. 3) more slowly for stronger fields. Lower plot in Fig. 3 displays also the spin expectation values $\left\langle\sigma_{x}\right\rangle$ and $\left\langle\sigma_{y}\right\rangle$ when RIC is not taken into account (dashed lines). As we will see later, the effect of RIC in higher subbands is more notable but we emphasize that even in the lowest subband the effect of Rashba intersubband 


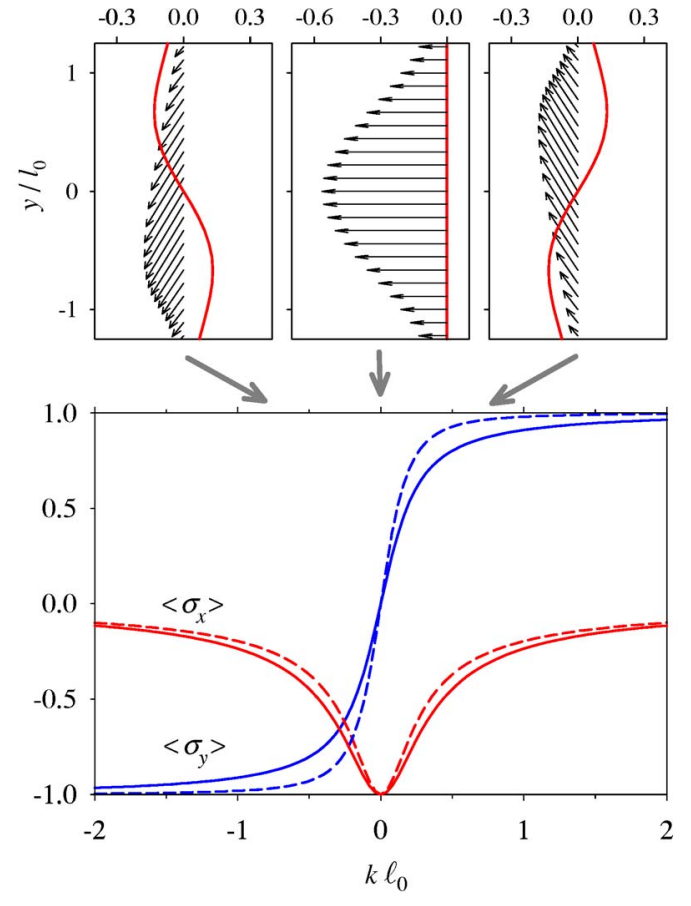

FIG. 3. (Color online) Lower plot: dependence with $k$ of the spin expectation values in the lowest subband. Solid lines are obtained including both Rashba terms while dashed lines are the results when RIC is neglected. We set $l_{Z}=2.2 l_{0}, l_{1}=l_{2}=l_{0}$, and $\theta=0$. Upper panels display the spin texture for three selected propagation momenta and taking into account both Rashba terms. The $k l_{0}$ values (indicated by the thick arrows pointing on the $k l_{0}$ axis) are $-0.75,0$, and 0.75 for left, center and right upper plots, respectively. The vector plot shows the in-plane spin and the solid line corresponds to the $z$ component.

coupling is not negligible due to the observation of spin textures.

We plot in Fig. 4 the spatially dependent magnetization and the spin expectation values for the second subband with the same parameters as in Fig. 3. The effect of RIC is more pronounced than in the lowest subband as seen from the spin textures, which contain a noncollinear distribution even for the in-plane spin. In addition the local $z$ magnetization is bigger than in the lower subband and exceeds the horizontal components, leading to large spin accummulations at the wire edges. For $k=0$ we again find that all local spins are properly aligned in the direction of transport. Remarkably, the spin expectation values $\left\langle\sigma_{x}\right\rangle$ and $\left\langle\sigma_{y}\right\rangle$ change drastically when the Rashba interaction is fully included as shown in lower Fig. 4. When RIC is absent, both expectation values display abrupt jumps due to the subband crossings. As we pointed out earlier, RIC avoids these crossings and this is reflected in a much smoother behavior of $\left\langle\sigma_{x}\right\rangle$ and $\left\langle\sigma_{y}\right\rangle$.

Opposite-sign accumulations of the $z$ magnetization at the wire edges are already found in the $B=0$ case. ${ }^{19,20}$ Differences with respect to the zero-field case, however, can be summarized in that the magnetic field causes nonzero $x$ magnetization across the wire for wave vectors around the minimum gap point. The tilted distributions of in-plane magnetizations in Fig. 3 are due entirely to the $x$ component of the field. Additionally, the texturing of the horizontal magnetiza-

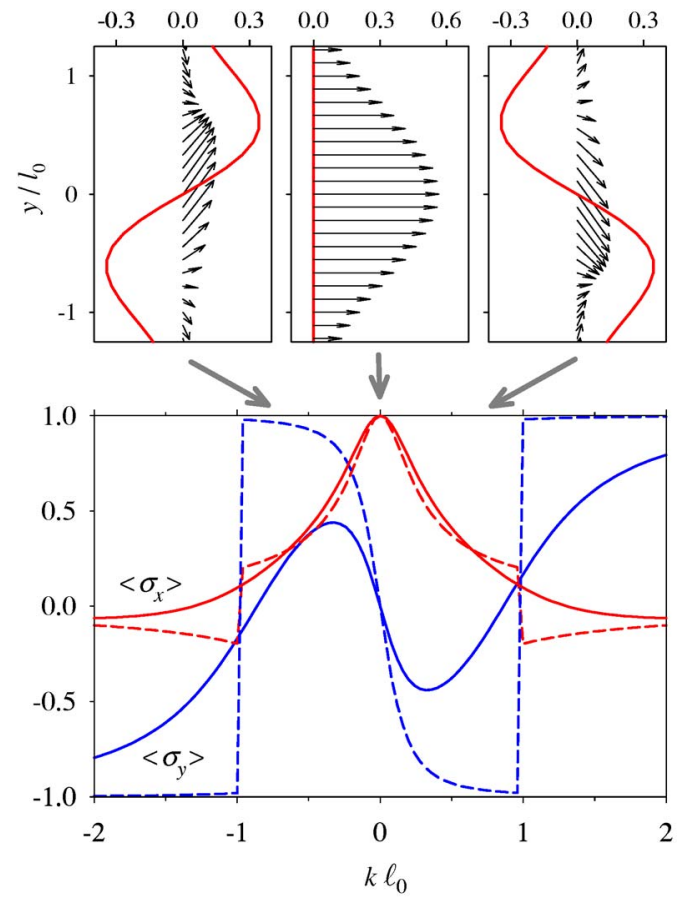

FIG. 4. (Color online) Same as Fig. 3 for the second subband.

tion of the second subband (Fig. 4) is due to the combination of $B$ and RIC. The magnetic field also shifts the minimum gap momentum $k_{g}$ from its zero value at vanishing $B$, for which an exact solution was obtained in the preceding section.

\section{TRANSPORT PROPERTIES}

The linear conductance $\mathcal{G}$ at zero temperature is directly related to the wire subband structure through the formula ${ }^{9,11}$

$$
\mathcal{G}=\frac{e^{2}}{h} \sum_{i j} \Theta\left(\mu-\xi_{i}^{(j)}\right) \operatorname{sgn}\left(m_{i}^{(j) *}\right),
$$

which accounts for the number of occupied subbands propagating in a given direction and the conductance quantum per spin component, $\mathcal{G}_{0}=e^{2} / h$. In Eq. (11), $\mu$ is the Fermi energy while $\xi_{i}^{(j)}$ and $m_{i}^{(j)^{*}}$ denote, respectively, the energy and effective mass at the $j$ th local extremum in the $i$ th subband. We have also defined $\Theta(x)$ and $\operatorname{sgn}(x)$ as the step and sign functions. For subbands containing a single minimum $\mathcal{G}$ only attains $e^{2} / h$ steps when $\mu$ is increased. In the case of more complicated subbands it is easy to see from Eq. (11) that the linear conductance step is doubled whenever the Fermi energy exceeds two local minima but is below the energy of the maximum lying in between them. Once $\mu$ exceeds the energy of the maximum, the conductance is decreased by $e^{2} / h .^{8,11}$ As discussed above RIC can convert subband maxima into minima and, therefore, $\mathcal{G}$ is generally reduced when both terms are included.

For $\theta=0$ we plot in Fig. 5 the linear conductance for the cases with RIC, $l_{2}=l_{1}$ (upper panel), and without it, $l_{2}=\infty$ (lower panel). When RIC is not included, $\mathcal{G}$ alternates, with 


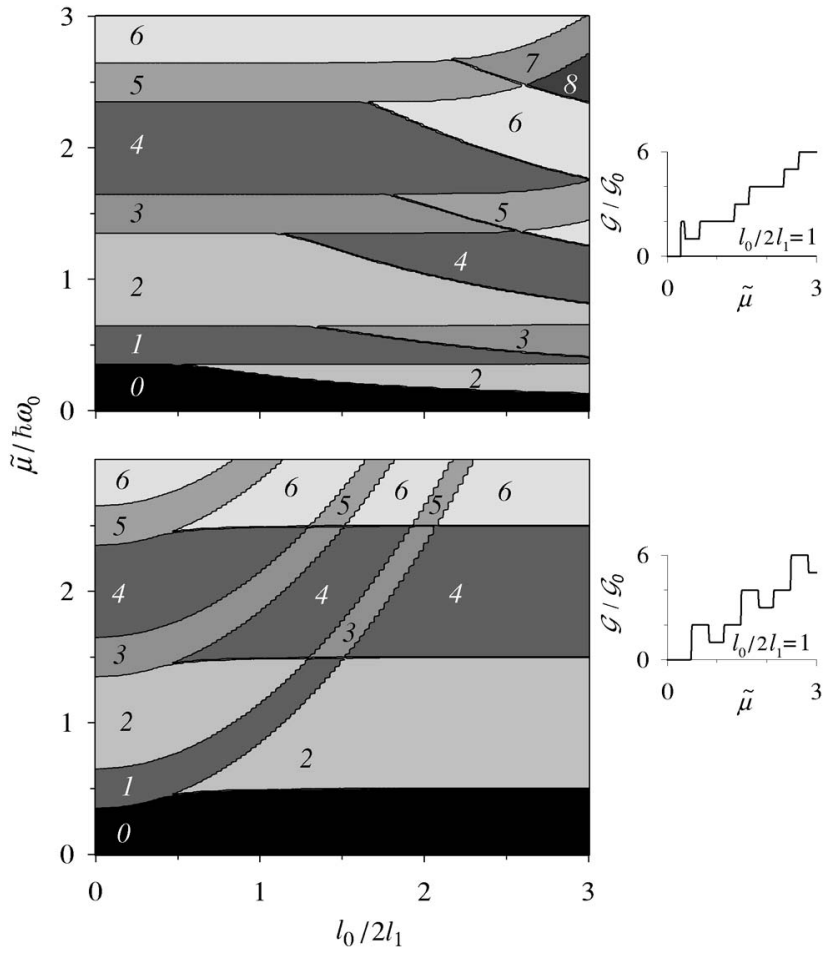

FIG. 5. Variation of the conductance with Rashba intensity and the Fermi energy $\mu$ in the full Rashba model $\left(l_{2}=l_{1}\right.$, upper panel) and neglecting RIC $\left(l_{2}=\infty\right.$, lower panel). We set $l_{Z}=1.8 l_{0}$ and parallel magnetic field $\theta=0$. A rigid shift with Rashba intensity has been taken into account defining $\tilde{\mu} / \hbar \omega_{0}=\mu / \hbar \omega_{0}+l_{0}^{2} / 8 l_{1}^{2}$. The numbers in the plateaus give the conductance in units of the conductance quantum. Right small plots show vertical cuts of the corresponding left figures for the given values of $l_{0} / 2 l_{1}$.

increasing $\mu$, between steps of $+2 e^{2} / h$ and downward jumps of $-e^{2} / h$ due to the presence of maxima in the lower subbands. ${ }^{11}$ When RIC is included two major modifications are apparent: (a) Stronger Rashba couplings are needed to observe the alternate steps of $+2 e^{2} / h$ and $-e^{2} / h$, and (b) in any case this anomalous pattern of steps vanishes when increasing the Fermi energy. Particularly, as shown by the insets, when $l_{0} / 2 l_{1}=1$ the sequence of conductance steps in units of $\mathcal{G}_{0}$ is $+2,-1,+1,+2,-1,+1, \ldots$ when RIC is neglected and $+2,-1,+1,+1,+1, \ldots$ when it is included. Therefore, Fig. 5 proves that to observe the modifications of the linear conductance steps due to the Rashba interaction it is essential to have a relatively low Fermi energy or, equivalently, a rather small number of propagating modes.

Figure 6 shows the case $\theta=\pi / 2$. In the lower part of Fig. 6 , when $l_{2}=\infty$, the conductance consists of regular jumps of one conductance quantum. From Fig. 2(b) we determine that the width of the odd conductance plateaus corresponds to the Zeeman energy whereas the width of the even plateaus amounts to $1-\left(l_{0} / l_{Z}\right)^{2}$ (in units of $\left.\hbar \omega_{0}\right)$. This physical scenario is strongly modified when RIC is taken into account, as seen in the upper Fig. 6 where the conductance shows a much richer behavior. The occurrence of restoration of the spin degeneracy for special values of the Rashba coupling and magnetic field where the third and fifth conductance

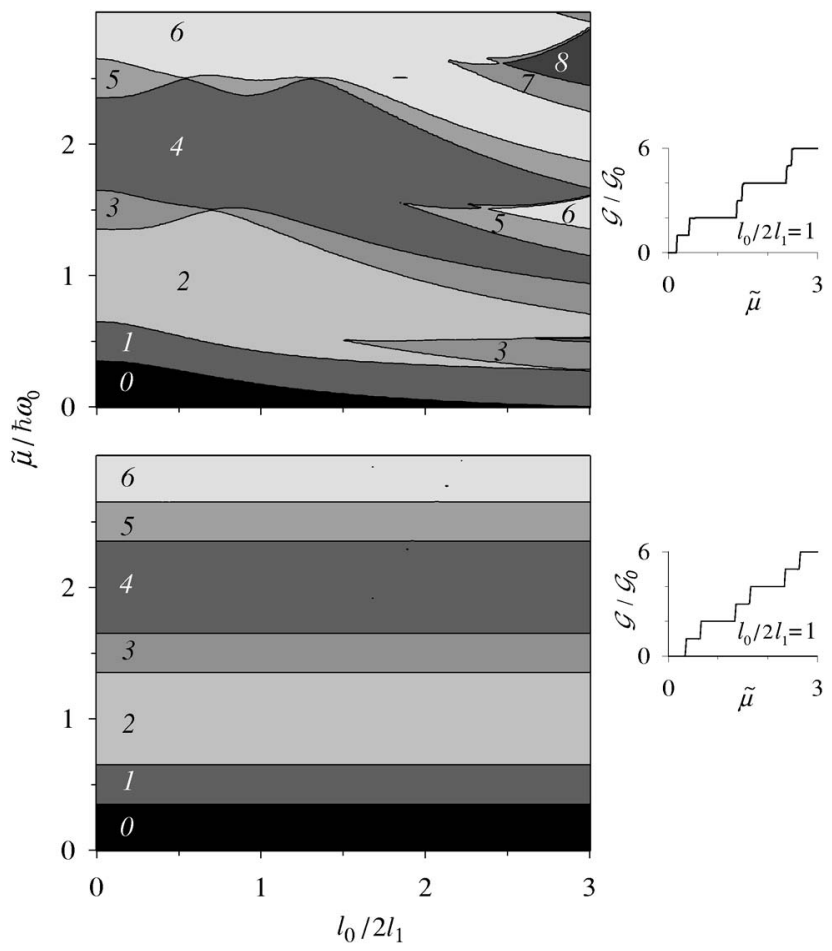

FIG. 6. Same as Fig. 5 for a perpendicular field $(\theta=\pi / 2)$.

plateaus collapse is remarkable. In general, the tendency is to suppress regions for $o d d$ values of $\mathcal{G}$. Thus, $\mathcal{G}$ presents wide (narrow) plateaus for even (odd) values of the conductance quantum but their widths are not trivially related to either the Zeeman energy or the confinement energy alone. Besides, at large Rashba strengths we also find decreasing jumps in the conductance.

\section{CONCLUSIONS}

We have discussed the spectrum, spin orientation, and linear transport of ballistic quantum wires in the presence of Rashba interactions and in-plane magnetic fields at an angle $\theta$ with the propagation direction. Analytical and numerical results demonstrating the crucial importance of the Rashba intersubband coupling for all these properties and for $k$ values near the subband gaps have been presented. At strong spin-orbit coupling the modifications in the energy subbands include the appearance of sizable shifts and anticrossings as well as big reductions of the subband maxima. The changes in subband structure lead to precise predictions for measurements of the linear transport properties, such as a severe reduction of the anomalous conductance steps when $\theta=0$ and a nontrivial dependence of the steps with the Rashba intensity for $\theta=\pi / 2$, with collapsing points for the odd plateaus.

Spin textures in the local magnetization are obtained only when the Rashba intersubband coupling is taken into account. Opposite-sign accumulations of the spin $z$ magnetization at the wire edges are strongly dependent on the $k$ value and subband index. For subbands above the lowest one we have shown that the horizontal magnetization is also textured. At a particular momentum (the minimum gap momen- 
tum), depending on magnetic-field strength, orientation, and Rashba intensity, the analytical solution having the spin along the transport direction has been obtained. We believe these results are relevant for the future design of spin transistors and spin filters.

\section{ACKNOWLEDGMENTS}

This research was supported by Spanish Grant Nos. PRIB-2004-9765 (Govern de les Illes Balears) and BFM2002-03241 (MEC) and the "Ramón y Cajal" program.
${ }^{1}$ E. I. Rashba, Fiz. Tverd. Tela (Leningrad) 2, 1224 (1960) [Sov. Phys. Solid State 2, 1109 (1960)].

${ }^{2}$ J. Nitta, T. Akazaki, H. Takayanagi, and T. Enoki, Phys. Rev. Lett. 78, 1335 (1997).

${ }^{3}$ G. Engels, J. Lange, Th. Schäpers, and H. Lüth, Phys. Rev. B 55, R1958 (1997).

${ }^{4}$ Th. Schäpers, J. Knobbe, and V. A Guzenko, Phys. Rev. B 69, 235323 (2004).

${ }^{5}$ S. Datta and B. Das, Appl. Phys. Lett. 56, 665 (1990).

${ }^{6}$ P. Streda and P. Seba, Phys. Rev. Lett. 90, 256601 (2003).

${ }^{7}$ J. C. Egues, G. Burkard, and D. Loss, Phys. Rev. Lett. 89, 176401 (2002).

${ }^{8}$ J. A. Nesteroff, Yu. V. Pershin, and V. Privman, Phys. Rev. Lett. 93, 126601 (2004).

${ }^{9}$ A. V. Moroz and C. H. W. Barnes, Phys. Rev. B 60, 14272 (1999).

${ }^{10}$ F. Mireles and G. Kirczenow, Phys. Rev. B 64, 024426 (2001).

${ }^{11}$ Yu. V. Pershin, J. A. Nesteroff, and V. Privman, Phys. Rev. B 69, 121306(R) (2004).
${ }^{12}$ M. Cahay and S. Bandyopadhyay, Phys. Rev. B 69, 045303 (2004).

${ }^{13}$ R. G. Pereira and E. Miranda, Phys. Rev. B 71, 085318 (2005).

${ }^{14}$ Yu. A. Bychkov, V. I. Mel'nikov, and E. I. Rashba, Zh. Eksp. Teor. Fiz. 98, 717 (1990) [Sov. Phys. JETP 71, 401 (1990)].

${ }^{15}$ S. Debald and B. Kramer, Phys. Rev. B 71, 115322 (2005).

${ }^{16}$ If $g<0$, the global sign of the Zeeman term should be reversed.

${ }^{17}$ G.-H. Chen and M. E. Raikh, Phys. Rev. B 60, 4826 (1999).

${ }^{18}$ M. Valín-Rodríguez, A. Puente, and Ll. Serra, Eur. Phys. J. B 34, 359 (2003).

${ }^{19}$ This result generalizes the $B=0$ case recently discussed by $\mathrm{M}$. Governale and U. Zülicke, Solid State Commun. 131, 581 (2004).

${ }^{20}$ M. Governale and U. Zülicke, Phys. Rev. B 66, 073311 (2002).

${ }^{21}$ J. Sinova, D. Culcer, Q. Niu, N. A. Sinitsyn, T. Jungwirth, and A. H. MacDonald, Phys. Rev. Lett. 92, 126603 (2004).

${ }^{22}$ G. Usaj and C. A. Balseiro, Europhys. Lett. 72, 631 (2005). 